\title{
Short communication: carboxylate functionalized superparamagnetic iron oxide nanoparticles (SPION) for the reduction of S. aureus growth post biofilm formation
}

\author{
This article was published in the following Dove Press journal: \\ International Journal of Nanomedicine \\ 19 February 2013 \\ Number of times this article has been viewed
}

\section{Kohana D Leuba \\ Naside Gozde Durmus \\ Erik N Taylor \\ Thomas J Webster}

The Nanomedicine Laboratory, School of Engineering, Brown University, Providence, RI, USA
Correspondence: Thomas J Webster Department of Chemical Engineering, 313 A Snell Engineering Center, 360 Huntington Avenue, Northeastern University, Boston, MA 02115, USA

Tel +617-373-6585

Fax +617-373-2209

Email th.webster@neu.edu

\begin{abstract}
Biofilms formed by antibiotic resistant Staphylococcus aureus (S. aureus) continue to be a problem for medical devices. Antibiotic resistant bacteria (such as S. aureus) often complicate the treatment and healing of the patient, yet, medical devices are needed to heal such patients. Therefore, methods to treat these biofilms once formed on medical devices are badly needed. Due to their small size and magnetic properties, superparamagnetic iron oxide nanoparticles (SPION) may be one possible material to penetrate biofilms and kill or slow the growth of bacteria. In this study, SPION were functionalized with amine, carboxylate, and isocyanate functional groups to further improve their efficacy to disrupt the growth of $S$. aureus biofilms. Without the use of antibiotics, results showed that SPION functionalized with carboxylate groups (followed by isocyanate then amine functional groups then unfunctionalized SPION) significantly disrupted biofilms and retarded the growth of $S$. aureus compared to untreated biofilms (by over 35\% after 24 hours).
\end{abstract}

Keywords: antibacterial, medical device infection, nanoparticle, iron oxide, biofilm, S. aureus

\section{Introduction}

An undesirable effect of inserting implants into the body to improve human health is infection. The most commonly reported medical device infections are associated with joint prosthesis, central venous catheters, endotracheal tubes, and mechanical heart valves. ${ }^{1}$ Often these infections can be frequent as well as costly depending on device location. ${ }^{1}$ The prevalence of infection by antibiotic resistant bacterial strains has risen considerably and is commonly seen in developed countries such as the United States. In particular, pathogenic bacteria, such as Staphylococcus aureus (S. aureus), are known to produce antibiotic resistant biofilms. Studies have shown that S. aureus currently accounts for at least $15 \%$ of all health care acquired infections. ${ }^{2}$ Shockingly, the multidrug resistant S. aureus (MRSA) has been reported in 56\% of all medical device related infections. ${ }^{2}$ Therefore, a method to overcome infection is badly needed since clearly our current attempts of developing antibiotics are not working.

Nanotechnology provides numerous materials with dimensions less than $100 \mathrm{~nm}$ that have found numerous applications in medicine and may penetrate biofilms and bacteria cell walls. ${ }^{3-22}$ In particular, due to their magnetic properties and location in the body which can be controlled externally through an applied electric field, superparamagnetic iron oxide nanoparticles (SPION) have a strong potential to disrupt biofilms 
produced by MRSA. ${ }^{4-6,19}$ These iron oxide nanoparticles (which can be in the form of maghemite, $\gamma-\mathrm{Fe}_{2} \mathrm{O}_{3}$ or magnetite, $\mathrm{Fe}_{3} \mathrm{O}_{4}$ ) are materials that can control drug delivery via an external magnetic source, the ability of which can be further improved by functionalizing their surface with molecules or chemistries to better penetrate biofilms and bacteria. In a number of in vivo experiments, SPION with functionalized surface chemistries have been used to enhance magnetic resonance imaging contrast, tissue repair, drug delivery as well as cell separation. ${ }^{21,22}$ SPION could be injected surrounding a biofilm and then an external magnet source could be used to draw the nanoparticles into the biofilm to disrupt bacteria functions. Recently, many studies have also demonstrated the use of magnetic particles coated with antibiotics as an alternative method for drug delivery, albeit such approaches still rely on the use of antibiotics for which bacteria are increasingly developing resistance towards. ${ }^{12,17}$

In this study, superparamagnetic iron oxide nanoparticles (SPION) were functionalized with amine, carboxylic acid, or isocyanate groups and, for the first time, were tested for their ability to penetrate $S$. aureus biofilms to explore a novel non-antibiotic strategy to decrease bacteria growth after a biofilm is formed on a medical device.

\section{Methods and materials $\mathrm{Fe}_{3} \mathrm{O}_{4}$ synthesis}

SPION were synthesized in a high temperature synthesis method in triethylene glycol. ${ }^{14} 2 \mathrm{mmol}$ of iron (III) acetylacetonate (Strem Chemicals, Newburyport, MA, USA) was dissolved in $25 \mathrm{~mL}$ of triethylene glycol (Alfa Aesar, Ward Hill, MA, USA) in a round bottom boiling flask with magnetic stirring. This solution was heated gradually to $278^{\circ} \mathrm{C}$ and the solution was refluxed at this temperature for 30 minutes. Nanoparticles were precipitated in $200 \mathrm{~mL}$ of $100 \%$ ethanol saturated with ammonium sulfate (SigmaAldrich, St Louis, MO, USA) and subsequently isolated in the presence of a strong magnetic field produced by two DZX08-N52 neodymium magnets (4" diameter $\times 1 / 2$ " thick magnet, 1795 Gauss; K\&J Magnetics, Jamison, PA, USA). Once SPION were precipitated from solution, the supernatant was decanted and particles were transferred to a $50 \mathrm{~mL}$ BD Falcon centrifuge tube, rinsed twice in $100 \%$ ethanol and separated from the ethanol using a neodymium block magnet $\left(4^{\prime \prime} \times 11^{\prime \prime} \times 1\right.$ ", 4871 Gauss; K\&J magnetics, Jamison, PA, USA). A sample of the solution was dried and the mass calculated. SPION were stored under refrigeration $\left(4^{\circ} \mathrm{C}\right)$ in a $100 \%$ ethanol solution, where no observable oxidation was seen in a period of less than a month.

\section{Amine functionalized SPION}

Using SPION created with the aforementioned high temperature synthesis in triethylene glycol, amine functionalized SPION were fabricated using a condensation reaction to create hydroxyl groups on the surface of the iron oxide nanoparticles. ${ }^{11}$ For this, 4 mmol of aminopropyltriethoxysilane (Sigma Aldrich) was added to the SPION and allowed to react overnight. SPION functionalized with amines (Figure 1A) on their surfaces were rinsed twice the next day in tetrahydrofuran (THF) using a neodymium block magnet as described in the washing steps of the uncoated SPION. The SPION was stored under refrigeration $\left(4^{\circ} \mathrm{C}\right)$ in a THF solution where no observable oxidation was seen in a period of less than a month.

\section{Carboxylate functionalized SPION}

Carboxylate functionalized SPION were prepared in a reaction with succinic anhydride (Sigma-Aldrich) and the amine functionalized SPION in a THF solution. A final $1 \%$ solution of succinic anhydride was used. ${ }^{17}$ This solution was allowed to react overnight and was rinsed twice in THF the next day to remove any unreacted succinic anhydride. These carboxylate coated SPION (Figure 1C) were stored under refrigeration in a THF solution where no observable oxidation was seen in a period of less than a month.

\section{Isocyanate functionalized SPION}

Similarly, the isocyanate functionalized SPION were prepared by using a new batch of SPION created using the aforementioned high temperature synthesis in triethylene glycol. These SPION were rinsed twice in THF to avoid the hydrolysisdecarboxylation reaction of the isocyanate in water. ${ }^{23} \mathrm{In}$ a condensation reaction with the hydroxyl groups on the surface, $4 \mathrm{mmol}$ of (3-isocyanatopropyl) triethoxysilane

A
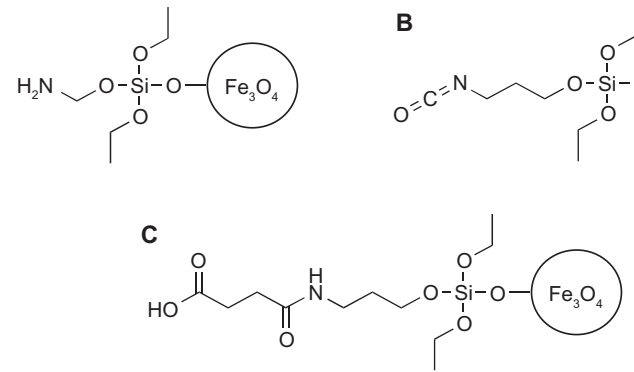

Figure I The (A) amine and (B) isocyanate functionalized SPION were prepared using silane chemistry. In a condensation reaction with the hydroxyl groups on the surface of the SPION, aminopropyl triethoxysilane was used to prepare the amine functionalized SPION and isocyanatopropyl triethoxysilane was used to prepare the isocyanate functionalized SPION. The (C) carboxylate functionalized SPION was prepared using the (A) amine functionalized SPION and succinic acid. 
(Sigma-Aldrich) was added to the SPION and allowed to react overnight. SPION functionalized with the isocyanate on the surface (Figure 1B) were rinsed twice the next day in THF using a neodymium block magnet as described in the washing steps of the uncoated SPION. These SPION were stored under refrigeration in a THF solution where no observable oxidation was seen in a period of less than a month.

\section{SPION characterization}

SPION morphology was visualized using transmission electron microscopy (TEM). Dispersions of SPION were dried on carbon coated copper grids. Samples were imaged by a Philips JOEL 140 kV TEM (Philips, New York, NY, USA). Moreover, inductively coupled plasma atomic emission spectroscopy (ICP-AES) was used to quantitatively confirm the presence of chemistries in the SPION. Lastly, magnetization measurements were performed using vibrating sample magnetometry (VSM, LakeShore 7400; Chicago, IL, USA) at T = $300 \mathrm{~K}$. To prepare samples for VSM, SPION were centrifuged and the supernatant was discarded. Then, nanoparticles were dispersed in ethanol, centrifuged again and the supernatant was discarded. Isolated nanoparticles were dried at room temperature for several days. Powders of nanoparticles were weighed and then placed in a capsule for hysteresis experiments at $\mathrm{T}=300 \mathrm{~K}$.

\section{Bacteria assays}

S. aureus (ATCC number 25923, American Type Culture Collection, Mannassas, VA, USA) was hydrated in tryptic soy broth (TSB) (MP Biomedicals, Solon, OH, USA) and streaked for isolation on a tryptic soy agar plate. After growth, a single isolated colony was selected and inoculated in $3 \mathrm{~mL}$ of TSB media. The bacteria culture was grown on an incubator shaker for 18 hours at $37^{\circ} \mathrm{C}, 200 \mathrm{rpm}$ until it reached stationary phase.

The optical density of the overnight culture at $562\left(\mathrm{OD}_{562}\right)$ was adjusted to 0.52 , which corresponds to $10^{9}$ cells. $^{22}$ The cultures were diluted $1: 10$ in TSB and $100 \mu \mathrm{L}$ was seeded into a 96-well plate (BD Biosciences, Franklin James, NJ, USA). Biofilms were formed by allowing the bacteria to incubate
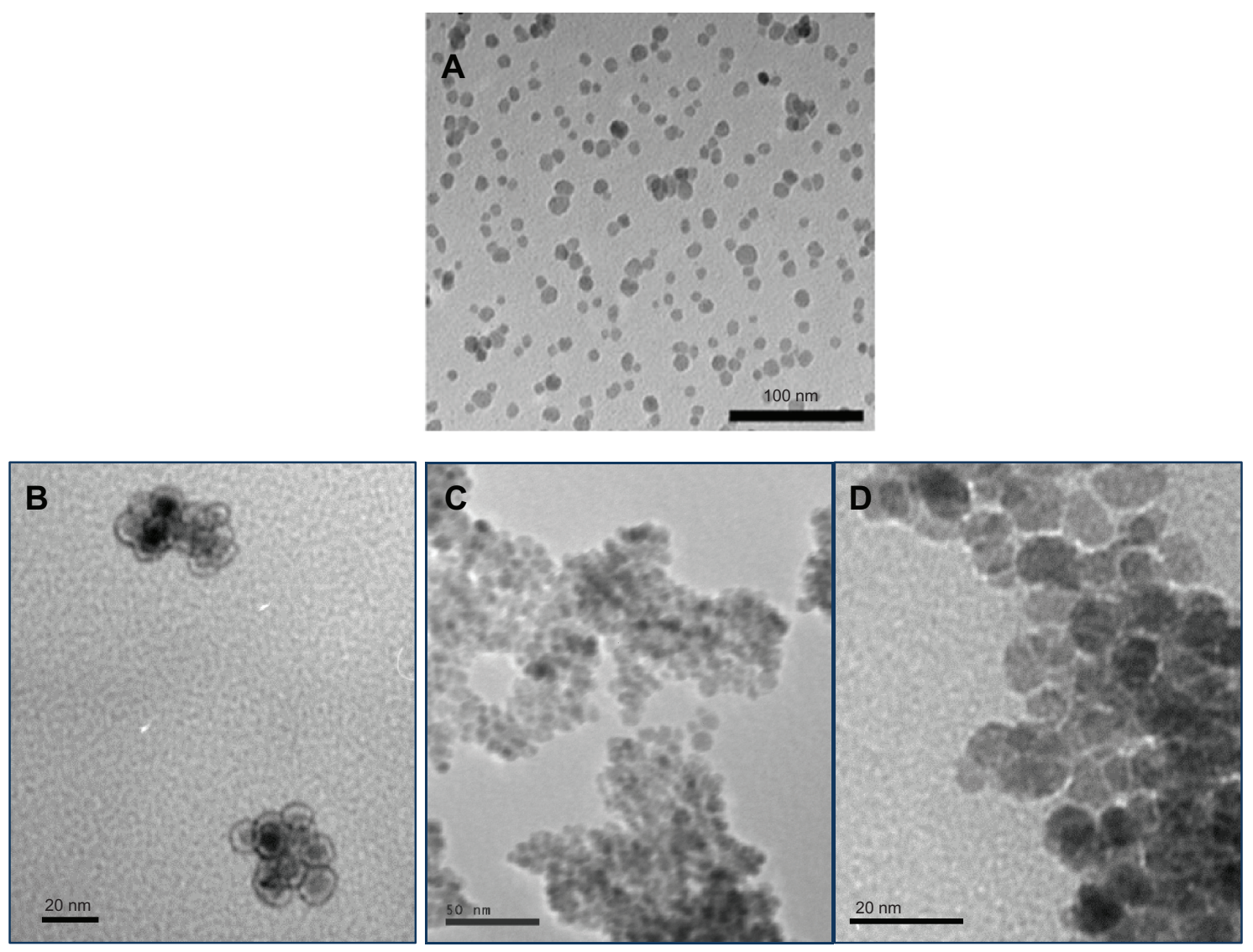

Figure 2 TEM images of $(\mathbf{A})$ unfunctionalized (scale bar $=100 \mathrm{~nm}),(\mathbf{B})$ amine functionalized, $(\mathbf{C})$ isocyanate functionalized, and (D) carboxylate functionalized SPION. Notes: Amine, isocyanate, and carboxylate functionalized SPION had an iron oxide core of $10-15 \mathrm{~nm}$ and a silicon coating of 3-4 nm. B (scale bar = $20 \mathrm{~nm}$ ), $C$ (scale bar $=50 \mathrm{~nm})$, and D (scale bar $=20 \mathrm{~nm}$ ).

Abbreviation: TEM, transmission electron microscopy. 
at $37^{\circ} \mathrm{C}$ for 24 hours. After incubation, each functionalized SPION was prepared by rinsing twice in phosphate buffered saline (PBS) using a neodymium block magnet and resuspended in PBS to reach a $1 \mathrm{mg} / \mathrm{mL}$ solution. $100 \mu \mathrm{L}$ of each SPION solution was added to each well with controls consisting of TSB alone. A SpectraMax MF plate reader (Molecular Devices, Sunnyvale, CA, USA) was used to analyze the change in bacterial growth over a period of 24 hours with OD measurements taken continuously every 4 minutes. The optical density readings of the controls were subtracted from each optical reading for the treated $S$. aureus samples.

\section{Statistical analysis}

All experiments were performed in triplicate with 8 replicates per experiment to validate repeatability and reproducibility. Differences between means were determined using ANOVA followed by student $t$-tests.

\section{Results and discussion \\ Material characterization}

TEM analysis indicated that all the SPION were between 10-20 nm in diameter (representative images for uncoated, amine coated and isocyanate coated SPION are shown in Figure 2). In addition, the silica coating on the amine functionalized SPION was detected from TEM images, as presented from the 3-aminopropyltriethoxysilane used during functionalization (Figure 2B). ICP-AES experiments demonstrated the expected chemistry of the various SPION formulated in the present study. Lastly, the magnetic moment for all the SPION was similar at $55 \pm 3$ amu.

\section{Bacteria studies}

Most importantly, Figure 3 shows the effect of SPION functionalization on $S$. aureus biofilms after 3, 10, and 24 hours. Clearly, the growth of $S$. aureus was slowed by

A

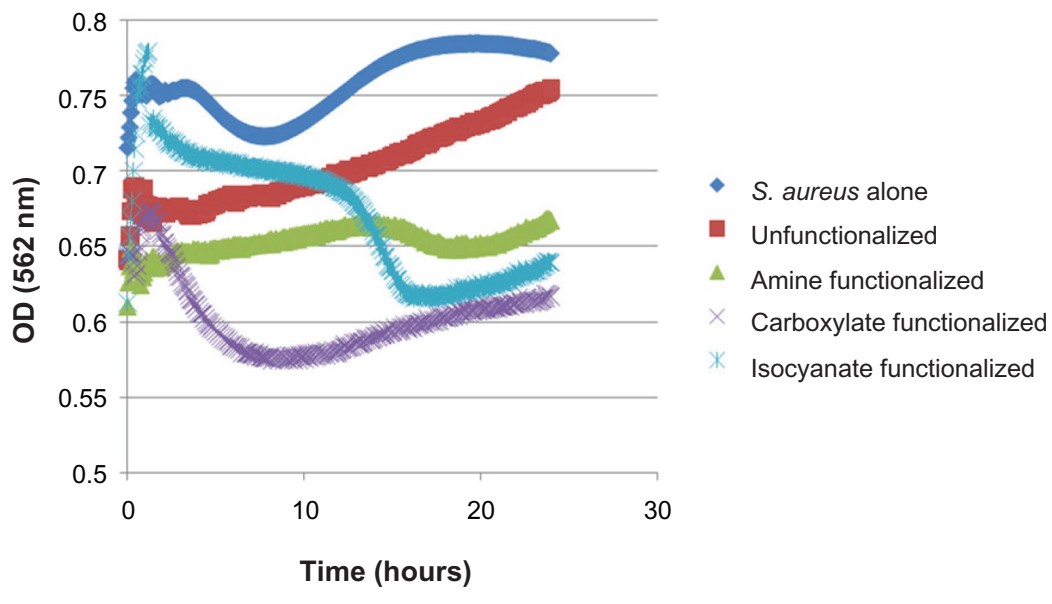

B

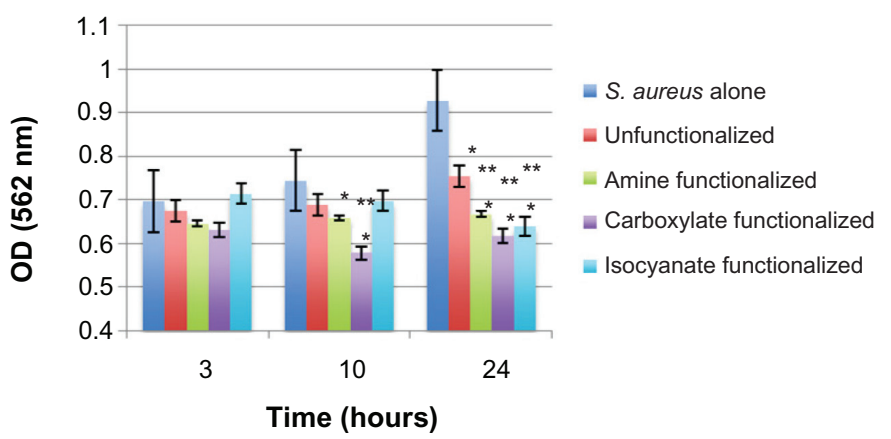

Figure 3 The effect of each type of SPION on S. aureus over the course of 24 hours with optical density measurements taken every 4 minutes. A) Raw data and B) select time periods of 3, 10, and 24 hours. Results of this experiment showed that the best SPION for treating S. aureus biofilm growth was SPION functionalized with carboxylate as well as iscoyanate. Data represent mean \pm SEM, $\mathrm{n}=3$ with $* P<0.1$ indicating statistical significance compared to $S$. aureus alone and $* * P<0.1$ indicating statistical significance compared to controls.

Notes: Results of this experiment showed that the best SPION for treating S. aureus biofilm growth was SPION functionalized with carboxylate as well as iscoyanate. Data represent mean $\pm S E M, n=3$ with $* P<0$.I indicating statistical significance compared to $S$. aureus alone and $* * P<0.1$ indicating statistical significance compared to controls (unfunctionalized).

Abbreviation: Unfunctionalized OD, optical density; SEM, Standard error of the mean. 
each functionalized SPION used in this study. Specifically, data from these experiments showed that the carboxylate functionalized SPION had the greatest impact on the growth of $S$. aureus after initial biofilm formation over the 24 hour time period. After the 24 hour period, carboxylate functionalized SPION had a $33.5 \%$ decrease in bacterial growth compared to the untreated $S$. aureus growth while the isocyanate, amine, and unfunctionalized SPION resulted in a $31.1 \%, 28.1 \%$, and $18.7 \%$ decrease, respectively. Thus, carboxyalate functionalized SPION were close to twice as potent at reducing bacteria function after biofilm formation than unfunctionalized SPION. It was also observed that the optical density increased after the lowest level was reached for the carboxyalate functionalized SPION. While the cause for this is currently unknown, more carboxyalate functionalized SPION particles may have been needed to provide additional anti-bacterial properties.

While requiring much more research, it is reasoned here that biofilm disruption and slowed bacteria growth occurred from SPION addition due to the increased uptake of nanoparticles by the biofilm and to the increased concentrations of iron in the biofilm and bacteria. Functionalizing SPION with carboxylate encouraged such biofilm and bacteria uptake. Recently, it was hypothesized that metabolite (fructose) enabled eradication of biofilms occurs through the catabolism of carbon sources and generation of nicotinamide adenine dinucleotide (NADH). ${ }^{22}$ Bacterial electron transport chain oxidizes NADH and contributes to a proton motive force. As a result, antibiotic uptake and the killing of persisters are facilitated. The same mechanism might take place during this study, as the SPION uptake may have also increased when functionalized with carboxylate. Then, bacterial death might occur due to oxidative stress since reactive oxygen species (ROS) formation increased in the presence of iron. It has been shown that SPION induce bacterial death through the formation of ROS. ${ }^{22}$ Therefore, first, superoxide $\left(\mathrm{O}^{2-}\right)$ formation could be stimulated by the electron chain transport and $\mathrm{O}^{2-}$ might damage iron clusters, making more ferrous iron available for oxidation by the Fenton reaction. The Fenton reaction leads to the formation of hydroxyl radicals $(\bullet \mathrm{OH})$, which damage DNA, lipids and proteins. All of these events eventually cause bacterial cell death and the eradication of biofilms.

In summary, while the mechanism that led to the decreased bacteria function by the presently fabricated functionalized SPION requires further investigation, these in vitro experiments indicated that such functionalized SPION should be further tested for a wide range of antibacterial applications.
The potential to treat $S$. aureus with SPION (and without the use of antibiotics) would make infection treatment less costly and not lead to the formation of antibiotic resistant bacteria.

\section{Conclusion}

Results of this study suggest a possible method to treat biofilm growth from $S$. aureus with SPION functionalized with carboxylate without resorting to the use of antibiotics. SPION functionalized with isocyanate, amine, and unfunctionalized SPION also did better at reducing in vitro biofilm growth than no treatment at all.

\section{Acknowledgments}

Special thanks to Mr Kyle A Totaro, Ms Emma Handy, and Dr Jason K Sello from the Department of Chemistry at Brown University for advice on chemical modification of iron oxide. The Karen T Romer Undergraduate Teaching and Research Award at Brown University funded this project.

\section{Disclosure}

The authors have nothing to disclose.

\section{References}

1. Donlan RM. Biofilms and device-associated infections. Emerg Infect Dis. 2001;7(2):277-281

2. Hidron AI, Edwards JR, Patel J, et al. Natl Healthcare Safety Network: Participating natl healthcare antimicrobial-resistant pathogens associated with healthcare-associated infections: Annual summary of data reported to the National Healthcare Safety Network at the Centers for Disease Control and Prevention, 2006-2007. Infect Control Hosp Epidemiol. 2008;29(11):996-1011.

3. Neu HC. The crisis in antibiotic resistance. Science. 1992;257(5073): 1064-1073.

4. Weissleder R, Bogdanov A, Neuwelt EA, Papisov M. Long-circulating iron oxides for MR-imaging. Adv Drug Deliv Rev. 1995;16(2-3):321-334.

5. Birch DG, Liang FQ. Age-related macular degeneration: a target for nanotechnology derived medicines. Int J Nanomedicine. 2007; 2(1):65-77.

6. Berry CC, Curtis ASG. Functionalisation of magnetic nanoparticles for applications in biomedicine. J Phys D-Appl Phys. 2003;36(13): R198-R206.

7. Taylor EN, Webster TJ. Reducing infections through nanotechnology and nanoparticles. Int J Nanomedicine. 2011;6:1463-1473.

8. Tripp RA, Alvarez R, Anderson B, Jones L, Weeks C, Chen W. Bioconjugated nanoparticle detection of respiratory syncytial virusinfection. Int J Nanomedicine. 2007;2(1):117-124.

9. Chen M, Yang Z, Wu H, Pan X, Xie X, Wu C. Antimicrobial activity and the mechanism of silver nanoparticle thermosensitive gel. Int $J$ Nanomedicine. 2011;6:2873-2877.

10. Tran PA, Webster TJ. Selenium nanoparticles inhibit Staphylococcus aureus growth. Int J Nanomedicine. 2011;6:1553-1558.

11. Čampelj S, Markovec D, Drofenik M. Functionalization of magnetic nanoparticles with 3-aminopropyl silane. Journal of Magnetism and Magnetic Materials. 2009;321:1346-1350.

12. Kell AJ, Steward G, Ryan S, et al. Vancomycin-modified nanoparticles for efficient targeting and preconcentration of gram-positive and gramnegative bacteria. ACS Nano. 2008;2:1777-1788. 
13. Beck JB, Killops KL, Kang T, et al. Facile preparation of nanoparticles by intramolecular crosslinking of isocyanate functionalized copolymers. Macromolecules. 2009;42(15):5629-5635.

14. Maity M, Kale SN, Kaul-Ghanekar R, Zue J, Ding J. Studies of magnetite nanoparticles synthesized by thermal decomposition of iron (III) acetylacetonate in tri(ethylene glycol). Journal of Magnetism and Magnetic Materials. 2009;321:3092-3098.

15. Brullot W, Reddy NK, Wouters J, et al. Versatile ferrorfluids based on polyethylene glycol coated iron oxide nanoparticles. Journal of Magnetism and Magnetic Materials. 2012;324:1919-1925.

16. Maurizi L, Bisht H, Bouyer F, Millot N. Easy route to functionalize iron oxide nanoparticles via long-term stable thiol groups. American Chemical Society, Langmuir Letter. 2009;25(16):8857-8859.

17. Kell AJ, Sinard B. Vancomycin architecture dependent on the capture efficiency of antibody-modified microbeads by magnetic nanoparticles. Chem Commun. 2007:1227-1229.
18. Machado MC, Cheng D, Tarquinio KM, Webster TJ. Nanotechnology: pediatric applications. Pediatr Res. 2010;67(5):500-504.

19. Tran P, Webster TJ. Enhanced osteoblast adhesion on nanostructured selenium compacts for anti-cancer orthopedic applications. Int $J$ Nanomedicine. 2008;3(3):391-396.

20. Puckett SD, Taylor E, Raimondo T, Webster TJ. The relationship between the nanostructure of titanium surfaces and bacterial attachment. Biomaterials. 2010;31(4):706-713.

21. Hu L, Percheron A, Chaumon D, Brachais CH. Microwave synthesis of core-shell structured biocompatible magnetic nanohybrids in aqueous medium; Microwave Heating; Usha Chandra (Ed): France; 2011:271.

22. Durmus NG, Taylor EN, Inci F, Kummer K, Tarquinio KM, Webster TJ. Fructose-enhanced reduction of bacterial growth on nanorough surfaces. Int J Nanomedicine. 2012;7:537-545.
International Journal of Nanomedicine

\section{Publish your work in this journal}

The International Journal of Nanomedicine is an international, peerreviewed journal focusing on the application of nanotechnology in diagnostics, therapeutics, and drug delivery systems throughout the biomedical field. This journal is indexed on PubMed Central, MedLine, CAS, SciSearch $\AA$, Current Contents ${ }^{\circledR} /$ Clinical Medicine,

\section{Dovepress}

Journal Citation Reports/Science Edition, EMBase, Scopus and the Elsevier Bibliographic databases. The manuscript management system is completely online and includes a very quick and fair peer-review system, which is all easy to use. Visit http://www.dovepress.com/ testimonials.php to read real quotes from published authors. 